\title{
Generalized algebra within a nonextensive statistics
}

\author{
L. Nivanen, A. Le Méhauté and Q.A. Wang \\ Institut Supérieur des Matériaux du Mans, \\ 44, Avenue F.A. Bartholdi, 72000 Le Mans, France \\ Email: awang@ismans.univ-lemans.fr
}

\begin{abstract}
By considering generalized logarithm and exponential functions used in nonextensive statistics, the four usual algebraic operators : addition, subtraction, product and division, are generalized. The properties of the generalized operators are investigated. Some standard properties are preserved, e.g., associativity, commutativity and existence of neutral elements. On the contrary, the distributivity law and the opposite element is no more universal within the generalized algebra.
\end{abstract}

PACS : 02.10.-v, 02.70.Rr, 02.90.+p

Keywords : Algebra, nonextensivity, generalized statistics

\section{Introduction}

Although scientists apply Boltzmann-Gibbs statistics (BGS), or its exponential factor of probability distribution, to systems having long range correlation or finite size[1], this classical statistical theory, from the usual point of view, remains an additive theory in the thermodynamic limits, i.e., the extensive thermodynamic quantities are proportional to its volume or to the number of its elements.

As far as we know, there is no direct experimental evidence of nonadditive energy. However, theoretical and numerical work has disclosed many models characterized by nonadditive energy or entropy with, e.g., black hole[2, 3] and some magnetic and fluid models including long range interaction $[4,5,6,7,8]$. Interesting numerical results also showed that, in the case of above long range (nonintegrable) interactions, in the thermodynamic limits, the systems show complex behaviors at the edge of chaos with non-gaussian distribution, anomalous diffusion and dynamic correlation in 
the phase space[9]. For this kind of non-equilibrium systems at stationary state[10], a possible way out has been suggested on the basis of a nonadditive statistical mechanics (NSM)[11, 12, 13, 14, 15, 16, 17, 18].

NSM introduces[11, 19] generalized distribution functions called $q$-exponential given by $\exp _{q}(x)=[1+(1-q) x]^{1 /(1-q)}$ or $\exp _{q}^{*}(x)=[1+(q-1) x]^{1 /(q-1)}$ according to different formalisms related to incomplete information[15, 16] and complete information hypothesis[11, 12, 13, 14]. The above functions are the inverse functions of the generalized logarithm $\ln _{q}(x)=\frac{x^{1-q}-1}{1-q}$ or $\ln _{q}^{*}(x)=\frac{x^{q-1}-1}{q-1}$ which can be used as a generalization of Hartley logarithmic information measure[15]. In NSM, we require that the [.] in $q$-exponential be positive to assure physically significant probability distribution. This requirement may lead to high or low energy cutoff depending on the value of $q$. In what follows, we will write the generalized functions as follows : $\ln _{a}(x)=\frac{x^{a}-1}{a}$ and $e_{a}^{x}=\exp _{a}(x)=[1+a x]^{1 / a}$. On the one hand, these forms are simpler to treat mathematically. On the other hand, this choice ensures the generality of the present results which should be independent of the physical circumstances with different formalisms of NSM. $e_{a}(x)$ and $\ln _{a}(x)$ tend to the conventional counterparts whenever $a \rightarrow 0$.

Although defined originally from the physical viewpoint, these functions present important mathematical interests. Some of their important properties have been studied recently in [20, 21]. They have also been used to generalize hyperbolic function and algebra[22]. In this paper, we discuss several properties of the new algebra generated by the $q$-logarithm and $q$-exponential. This algebra can be viewed as a generalization of the conventional additive algebra related to the normal logarithm and exponential, but characterized by a nonadditive additional factor. A similar generalization of the conventional algebra has been proposed by Kaniadakis on the basis of the $\kappa$-deformed logarithm and exponential[23].

The paper is organized as follows. In the following section we briefly discuss the essential aspects of NSM. The generalized algebra is discussed in the third section.

\section{Some nonadditive relations of NSM}

We know that, for a nonadditive thermodynamic system, the existence of thermodynamic stationarity may be used as a constraint upon the form of the nonextensivity of physical quantities[24]. For the entropy $S$ and the internal energy $U$, this can be discussed rigorously by using the conventional BGS method for the statistical interpretation of the zeroth law. It 
has been shown[24] that the simplest nonextensivities prescribed by the thermodynamic stationarity condition were generated by the following relationships : $S(A)=\frac{h(A)-1}{\lambda_{S}}, S(B)=\frac{h(B)-1}{\lambda_{S}}, S(A+B)=\frac{h(A+B)-1}{\lambda_{S}}$ and $h(A+B)=h(A) h(B)$, where $A$ and $B$ are two subsystems of an isolated system $A+B, \lambda_{S}$ is a constant, $h(A)$ or $h(B)$ is the factor depending on $A$ or $B$ in the derivative $\frac{\partial S(A+B)}{\partial S(B)}$ or $\frac{\partial S(A+B)}{\partial S(A)}$. It was also found that these relationships are also valid if $S$ is replaced by $U$ and $\lambda_{S}$ by $\lambda_{U}$ [25]. So the nonextensivities should be the following : $S(A+B)=S(A)+S(B)+\lambda_{S} S(A) S(B)$ and $U(A+B)=U(A)+U(B)+\lambda_{U} U(A) U(B)$. We have shown[16] that only these two relationships allow to interpret the zeroth law of thermodynamics within NSM and define a physical temperature without any approximation.

The above nonextensivity of entropy, according to some authors[26], uniquely leads to the entropy

$$
S=-k \frac{\sum_{i} p_{i}-\sum_{i} p_{i}^{q}}{1-q}
$$

which can be shown $[15,16]$ to be the following expectation value $S=\sum_{i} p_{i}^{q} I_{i}$ of a generalized information measure :

$$
I_{i}=k \ln _{1-q}\left(p_{i}\right)
$$

where $q=1+\lambda_{S}$ is a real parameter[11], $k$ is Boltzmann constant and $p_{i}$ the probability that the system is found at the state $i$, if it is supposed that $p_{i j}(A+B)=p_{i}(A) p_{j}(B)$. This product law of probability has always been considered as a result of the "statistical independence" of $A$ and $B$ and considered by many as a "reason" for accepting additive energy $U(A+B)=$ $U(A)+U(B)$ within NSM. As shown with the counter-example, this is not necessarily true and entails several fundamental and practical problems which have been discussed in detail[16]. We observed[27] that the product law of probability could be considered as a consequence of the entropy in Eq.(1) and the nonextensivity imposed by the condition of the existence of equilibrium. In this way, the composite energy is freed from the constraint of the independence of the subsystems and NSM is then entitled to treat nonadditive systems having the nonadditive entropy postulated in Eq.(1) (or the information measure postulated in Eq.(2) and the nonadditive energy prescribed by the thermal equilibrium.

The maximization of the above entropy leads to the $q$-exponential probability distribution. The currently improved coherence of this statistics and the investigations of the relationships between this statistics and the chaotic or fractal phenomena[28] tell us that $q$-logarithm and $q$-exponential have 
solid physical background and may play a major role in the nonadditive physics. So it is worthwhile to investigate them from pure mathematical point of view and especially according to the related algebra associated with nonadditive properties. In what follows, we present the generalized nonadditive algebra related to these functions.

\section{Generalized operations}

From now on, the mathematical notion of morphism will be used to exhibit generalized expressions for the four fundamental algebraic operators : addition, subtraction, product and division. In the fourth part, the reader will see that the algebraic properties of these generalized operators are not always identical to those of the classical operators. New features will be pointed out, for instance, the distributive law is no more verified.

The standard exponential and logarithm functions present some remarkable properties. For instance, the exponential function is a morphism from $(\mathbf{R},+)$ to $\left(\mathbf{R}_{+}^{*}, \times\right)$. Through reciprocity, the logarithm function is a morphism from $\left(\mathbf{R}_{+}^{*}, \times\right)$ to $(\mathbf{R},+)$. As a result : $e^{x+y}=e^{x} e^{y}$ and $\ln x y=$ $\ln x+\ln y$. Although these properties cannot be generalized directly by a simple substitution of $e^{x}$ by $e_{a}^{x}$ (or $\ln x$ by $\ln _{a} x$, the following slightly more complicated relationships are verified :

$$
\begin{gathered}
e_{a}^{x} e_{a}^{y}=e_{a}^{x+y+a x y} \neq e_{a}^{x+y}, \\
e_{a}^{x+y}=\left[\left(e_{a}^{x}\right)^{a}+\left(e_{a}^{y}\right)^{a}-1\right]^{1 / a} \neq e_{a}^{x} e_{a}^{y}, \\
\ln _{a} x+\ln _{a} y=\ln _{a}\left(x^{a}+y^{a}-1\right)^{1 / a} \neq \ln _{a} x y, \\
\ln _{a} x y=\ln _{a} x+\ln _{a} y+a \ln _{a} x \ln _{a} y \neq \ln _{a} x+\ln _{a} y .
\end{gathered}
$$

We see that with standard addition and product operators, the concept of morphism cannot be applied to generalized functions. But if we define generalized addition (denoted by $+_{a}$ ) and product $\left(\times_{a}\right)$ operators that depend on the parameter $a$ as follows :

$$
x+{ }_{a} y=x+y+a x y
$$

and

$$
x \times_{a} y=\left(x^{a}+y^{a}-1\right)^{1 / a},
$$


then Eqs.(3) to (6) can be recast into :

$$
\begin{gathered}
e_{a}^{x+a y}=e_{a}^{x} e_{a}^{y}, \\
e_{a}^{x+y}=e_{a}^{x} \times_{a} e_{a}^{y}, \\
\ln _{a} x \times_{a} y=\ln _{a} x+\ln _{a} y, \\
\ln _{a} x y=\ln _{a} x+{ }_{a} \ln _{a} y .
\end{gathered}
$$

The two standard morphisms have split into four ones. Let us denote the definition set of the function $e_{a}^{x}$ by $D_{a}$. From Eq.(9), it comes that the generalized exponential function is a morphism from $\left(D_{a},+_{a}\right)$ to $\left(\mathbf{R}_{+}^{*}, \times\right)$. But from Eq.(10), we note that this function is also a morphism from $\left(D_{a},+\right)$ to $\left(\mathbf{R}_{+}^{*}, \times_{a}\right)$. From Eq.(11), it comes that the generalized logarithm function is a morphism from $\left(\mathbf{R}_{+}^{*}, \times_{a}\right)$ to $\left(D_{a},+\right)$. But from Eq.(12), we note that this function is also a morphism from $\left(\mathbf{R}_{+}^{*}, \times\right)$ to $\left(D_{a},+_{a}\right)$. It is worth noticing that a standard operator and a generalized one are present in each of the Eqs.(9) to (12). This is because that the generalized operators are defined with the help of the standard ones in Eqs.(7) and (8).

In the same way, the generalized subtraction $-{ }_{a}$ and division $/ a$ operators can be defined. If we write :

$$
x-{ }_{a} y=\frac{x-y}{1+a y}
$$

and

$$
x /{ }_{a} y=\left(x^{a}-y^{a}+1\right)^{1 / a}
$$

then the following relationships exist :

$$
\begin{gathered}
e_{a}^{x-a y}=e_{a}^{x} / e_{a}^{y} \\
e_{a}^{x-y}=e_{a}^{x} /{ }_{a} e_{a}^{y} \\
\ln _{a}\left(x /{ }_{a} y\right)=\ln _{a} x-\ln _{a} y \\
\ln _{a}(x / y)=\ln _{a}(x)-{ }_{a} \ln _{a} y .
\end{gathered}
$$

which recover the standard ones $e^{x-y}=e^{x} / e^{y}$ and $\ln (x / y)=\ln x-\ln y$ when $a \rightarrow 0$. 


\section{Properties of generalized operators}

\subsection{Additivity}

The generalized addition operator has following properties :

1. Associativity : $\left(x+{ }_{a} y\right)+{ }_{a} z=x+{ }_{a}\left(y+{ }_{a} z\right)$.

2. Commutativity : $x+{ }_{a} y=y+{ }_{a} x$.

3. 0 is the neutral element, i.e., $x+{ }_{a} 0=0+{ }_{a} x=x$.

4. If $x \neq x_{0}=-1 / a$, it has an opposite element $-{ }_{a} x$, i.e., $-{ }_{a} x=$ $-x /(1+a x)$. The fact that $x_{0}$ has no opposite element is a new feature. It should be noticed that the above definition of opposite element is compatible with the definition of substraction : $x-{ }_{a} y=x+{ }_{a}\left(-{ }_{a} y\right)$.

5. The sign rules : $-{ }_{a}\left(-{ }_{a} x\right)=+{ }_{a}\left(+{ }_{a} x\right)=x$ and $-{ }_{a}\left(+{ }_{a} x\right)=+{ }_{a}\left(-{ }_{a} x\right)=$ $-{ }_{a} x$.

6. Generating role of $+_{1}$ : if we note $Z=x+{ }_{a} y$, then $a Z=(a x)+{ }_{1}(a y)$.

\subsection{Multiplication}

The product operator $\times_{a}$ has more complicated features. For a given $a$, we see from Eq.(5) that, if $x, y>0$, the product $x \times_{a} y$ is defined unambiguously and the inequality $x^{a}+y^{a}>1$ is verified. We notice following properties :

1. Associativity : $\left(x \times_{a} y\right) \times_{a} z=x \times_{a}\left(y \times_{a} z\right)$.

2. Commutativity : $x \times_{a} y=y \times_{a} x$.

3. 1 is the neutral element, i.e., $x \times{ }_{a} 1=1 \times_{a} x=x$.

4. There is no absorbing element (like zero in the usual case). We cannot find a real number $y$ such that, for arbitrary real number $x$, we have $x \times_{a} y=y$.

5. $\mathrm{x}$ has an inverse element noted $1 /{ }_{a} x=\left(2-x^{a}\right)^{1 / a}$. In particular, we see that if $a>0$, then $1 / a 0=2^{1 / a}$ and $1 / a 2^{1 / a}=0$. So 0 can have a finite inverse in the generalized algebra.

6. Generating role of $\times_{1}$ : if we note $Z=x \times_{a} y$, then $Z^{a}=\left(x^{a}\right) \times_{1}\left(y^{a}\right)$. 
We notice that the new operators of this generalized algebra have more complex properties than the usual ones. Unlike the standard case, the existence of an opposite element is not automatic. On the other hand, 0 can now be inverted. Now with the new operators, the infinity concept may be taken into account with finite numbers. There is a strong analogy between this property and the role of hyperbolic space in metric topology[29]. It is natural to find these two distinct mathematical tools in the study of physical phenomena taking place on complex media characterized by a set of singularities in a compact space[30, 31].

Another important feature of the new algebra with the operators defined previously is the disparition of the law of distributivity, i.e., $x y+x z=$ $x(y+z)$ within the usual algebra. With the above generalized operators, no combination is possible, except for a particular case like $\mathrm{x}=1$. It is straightforward to show that, in general, we have :

$$
\begin{gathered}
x \times_{a} y+x \times_{a} z \neq x \times \times_{a}(y+z) \\
x y+{ }_{a} x z \neq x\left(y+{ }_{a} z\right) \\
x \times_{a} y+{ }_{a} x \times_{a} z \neq x \times{ }_{a}\left(y+{ }_{a} z\right)
\end{gathered}
$$

This non distributivity has important consequences for the manipulation of analytical expressions. The most important one is the impossibility to develop or factorize expressions.

On the other hand, distributivity can be recovered by defining different generalized addition and product operators as has been done in [23] :

$$
\ln \left[e_{a}^{x \times{ }^{a} y}\right]=\ln e_{a}^{x} \ln e_{a}^{y}
$$

and

$$
e^{\left[\ln _{a}\left[x+{ }^{a} y\right]\right.}=e^{\ln _{a} x}+e^{\ln _{a} y} .
$$

The distributivity can be established with either $\times^{a}$ and $+_{a}$ or $\times_{a}$ and $+^{a}$, i.e. :

$$
x \times{ }^{a} y+{ }_{a} x \times{ }^{a} z=x \times \times^{a}\left(y+{ }_{a} z\right)
$$

or

$$
x \times{ }_{a} y+{ }^{a} x \times{ }_{a} z=x \times{ }_{a}\left(y+{ }^{a} z\right) .
$$

It should be noted that the distributivity does not exist neither between the $\times^{a}$ and $+{ }^{a}$ defined in Eqs.(22) to (23), i.e. :

$$
x \times{ }^{a} y+{ }^{a} x \times{ }^{a} z \neq x \times{ }^{a}\left(y+{ }^{a} z\right),
$$


nor between these two operators and the ordinary operators.

The operators $\times^{a}$ and $+{ }^{a}$ are respectively given by

$$
x \times{ }^{a} y=\frac{e^{\ln (1+a x) \ln (1+a y) / a}-1}{a}
$$

and

$$
x+{ }^{a} y=\left[a \ln \left(e^{x^{a} / a}+e^{y^{a} / a}\right)\right]^{1 / a},
$$

which are different from $\times_{a}$ and $+_{a}$ and have different properties. For example, we have here $x+{ }^{a} 0 \neq x, x \times \times^{a} 1 \neq x$ and $x \times{ }^{a} 0=0$.

\section{Examples of simple application}

It is obvious that, if we use this generalized algebra with the operators defined in Eqs.(7) to (12), the nonadditive statistical mechanics and thermodynamics will be able to be expressed in additive form just as in BGS. In one of our papers[18], NSM is given in additive form by deforming the nonadditive energy and entropy. The same formalism can be given with generalized algebra without deforming the nonadditive physical quantities. That is, for a total system composed of two subsystems $A$ and $B$, we can write for entropy $S(A+B)=S(A)+a_{S} S(B)$ and for energy $U(A+B)=U(A)+a_{U} U(B)$ where $a_{S}=-\lambda_{S}=(1-q) / k$ and $a_{U}=-\lambda_{U}=(1-q) / k T$ for NSM.

Let us consider the temporal evolution of the price of a product at successive 1st of January. It costs respectively $x_{0}=100 \$$ in $2001, x_{1}=110 \$$ in 2002 and $x_{2}=121 \$$ in 2003 . The annual evolution ratio is $y_{0}=\left(x_{1}-\right.$ $\left.x_{0}\right) / x_{0}=0,1$ and $y_{1}=\left(x_{2}-x_{1}\right) / x_{1}=0.1$, or $10 \%$ each time. The global evolution ratio over two years is $y_{0}^{\prime}=\left(x_{2}-x_{0}\right) / x_{0}=0.21$, that is $21 \%$. Of course $y_{0}^{\prime} \neq y_{0}+y_{1}$ because the ratios are not based upon the same

denominator. However, simple calculation leads to $y_{0}^{\prime}=y_{0}+y_{1}+y_{0} y_{1}$. This expression is rewritten with the help of the generalized addition operator defined in Eq.(7) : $y_{0}^{\prime}=y_{0}+{ }_{1} y_{1}$. It means that combinations of ratios can be considered as additive by using the generalized addition. In this case, $a=1$ is a universal value. This can be shown for whatever ratios as follows. From Eq. (7), we have $a=\left(\frac{x_{2}-x_{0}}{x_{0}}-\frac{x_{1}-x_{0}}{x_{0}}-\frac{x_{2}-x_{1}}{x_{1}}\right) / \frac{\left(x_{1}-x_{0}\right)\left(x_{2}-x_{1}\right)}{x_{0} x_{1}}=\frac{x_{1}-x_{0}}{x_{1}-x_{0}}=1$. This calculation can be useful for other temporal process.

\section{Conclusion}

In this paper some algebraic aspects of the nonadditive statistics have been studied. The four classical operators of the usual algebra have been gener- 
alized. The new operators allow to preserve the morphism properties of the exponential and logarithm functions. Some properties (associativity, commutativity, existence of neutral element and the sign rules) of the standard operators can be extended to the generalized ones. But others cannot, e.g. the opposite element does not exist for arbitrary element; and the generalized additions and multiplications are not always distributive. Another interesting point should be noted : in the generalized formalism, " 0 " is no more the absorbing element of multiplication but can be inverted just like other element.

We hope that this generalized algebra can be helpful for the understanding and the development of nonadditive physics which seems inevitable in

view of the complex phenomena that cannot be described within the additive statistical theory.

\section{References}

[1] J.L. Cardy, Conformal invariance and statistical mechanics, in Fields, strings and critical phenomena, Les Houches 1988, Ed. by E. Brzin and J. Zinn-Justin, North Horland (1990)p168

B. Jancovici, G. Manificat and C. Pisani, Coulomb systems seen as critical systems : finite-size effects in two dimensions, J. Stat. Phys., 70(1994) 3147

[2] Sean A. Hayward, Class. Quant. Grav., 15(1998)3147, gr-qc/9710089

Sean A. Hayward, Phys. Lett. A, 256(1999)347-350

[3] V.P. Frolov, D.V. Fursaev, and A.I. Zelnikov, Phys. Lett. B, 382(1996)220, Hep-th/9603175

[4] P. Jund, S.G. Kim and C. Tsallis, Phys. Rev. B, 52(1995)50

[5] S.A. Cannas and F.A. Tamarit, Phys. Rev. B, 54(1996)R12661

[6] M. Antoni and S. Ruffo, Phys. Rev. E, 52(1995)2361

[7] J.R. Grigera, Phys. Lett. A, 217(1996)47

[8] J. Barré, D. Mukamel and S. Ruffo, Phys. Rev. Lett., 87(2001)030601

J. Barré, D. Mukamel and S. Ruffo, Lecture Notes in Physics, 602(2002)45-67 
T. Dauxois, S. Ruffo, E. Arimondo, and M. Wilkens, Lecture Notes in Physics, 602(2002)1-19

[9] V. Latora, A. Rapisarda, C. Tsallis, Physica A, 305(2002)129

V. Latora, A. Rapisarda, C. Tsallis, Phys. Rev. E, 64(2001)056134

V. Latora, A. Rapisarda, S. Ruffo, Prog. Theor. Phys. Suppl., $\mathbf{1 3 9}(2000) 204$

[10] S. Abe, A.K. Rajagopal, A. Plastinos, V. Lotora, A. Rapisarda and A. Robledo, Letters to the Editor : Revisiting disorder and Tsallis statistics, Science, 300(2003)249-251

[11] C. Tsallis, J. Stat. Phys., 52(1988)479

[12] EMF. Curado and C. Tsallis, J. Phys.A:Math.Gen., 24 (1991)L69

[13] F. Pennini, A.R. Plastino and A. Plastino, Physica A, 258(1998)446

[14] C. Tsallis, R.S. Mendes and A.R. Plastino, Physica A, 261(1999)534; Silvio R.A. Salinas and C. Tsallis, Brazilian Journal of Physics(special issue: Nonadditive Statistical Mechanics and Thermodynamics), 29(1999).

[15] Q.A. Wang, Incomplete statistics : nonextensive generalization of statistical mechanics, Chaos, Solitons \& Fractals, 12(2001)1431

[16] Q.A. Wang, Nonextensive statistics and incomplete information, Euro. Phys. J. B, 26(2002)357

[17] Q.A. Wang, Correlated electrons and generalized statistics, Euro. Phys. J. $B, \mathbf{3 1}(2003) 75-79$

[18] Q.A. Wang and A. Le Méhauté, Extensive form of equilibrium nonextensive statistics, J. Math. Phys, 43(2002)5079-5089

[19] C. Tsallis, Quimica Nova, 17 (1994)468

[20] Takuya Yamano, Physica A, 305(2002)486

[21] C. E. Aguiar and T. Kodama, Physica A, 320(2003)371

[22] E.P. Borges, J. Phys.A:Math.Gen., 31 (1998)5281-5288;

E.P. Borges, A possible deformed algebra and calculus inspired in nonextensive thermostatistics, cond-mat/0304545 
[23] G. Kaniadakis, Phys. Rev. E, 66(2002)056125

G. Kaniadakis, Physica A, 296(2001)405

[24] S. Abe, Phys. Rev. E, 63(2001)061105

[25] Q.A. Wang, L. Nivanen, A. Le Méhauté and M. Pezeril, On the generalized entropy pseudoadditivity for complex systems, J. Phys. A, 35(2002)7003

[26] R.J.V. dos Santos, J. Math. Phys., 38(1997)4104

S. Abe, Phys. Lett. A, 271(2000)74

[27] Q.A. Wang, Phys. Lett. A, 300(2002)169

Q.A. Wang, Chaos, Solitons \& Fractals, 14(2002)765

[28] Q.A. Wang, Incomplete information in fractal phase space, Chaos, Solitons \& Fractals, (2003), in press, cond-mat/0207647

Q.A. Wang, Measuring information growth in fractal phase space, condmat/0305540

[29] A.F. Beardon, An introduction to hyperbolic geometry, in Ergodic theory, symbolic dynamics and hyperbolic spaces, Oxford University Press, New York, (1991)

[30] A. Le Méhauté, R. Nigmatullin, L. Nivanen, Flèches du temps et géométrie fractale, Hermes, Paris, (1998)

[31] A. Le Méhauté, L. Nivanen, Proceedings of SPIE, 4061(2000)180. 